\title{
Pulmonary flow study predicts survival in pulmonary atresia with ventricular septal defect and major aortopulmonary collateral arteries
}

Jiaquan Zhu, MD, PhD, , ,b James Meza, MD, a Atsuko Kato, MD, ${ }^{\mathrm{c}}$ Arezou Saedi, MD, Devin Chetan, MD, ${ }^{\mathrm{a}, \mathrm{d}}$ Rachel Parker, BScH, ${ }^{\mathrm{a}}$ Christopher A. Caldarone, MD, ${ }^{\mathrm{a}}$ Brian W. McCrindle, MD, MPH, ${ }^{\mathrm{b}}$ Glen S. Van Arsdell, MD, ${ }^{\mathrm{a}}$ and Osami Honjo, MD, PhD ${ }^{\mathrm{a}}$

\begin{abstract}
Background: We hypothesized that mean pulmonary artery pressure (PAP) detected on a pulmonary flow study may predict medium-term survival and right ventricular systolic pressure (RVSP) in patients with pulmonary atresia (PA), ventricular septal defect (VSD), and major aortopulmonary collateral arteries (MAPCAs).

Methods: Fifty patients with PA/VSD/MAPCAs underwent unifocalization between 2000 and 2013, and 40 of these patients had a pulmonary flow study since 2003. Predictability of the mean PAP on VSD status, medium-term survival, reintervention, and RVSP were analyzed.
\end{abstract}

Results: Forty-seven of the 50 patients $(94 \%)$ had complete unifocalization at a median age of 11 months (range, 1-194 months), and 37 patients (74\%) achieved VSD closure. Among the 40 patients who underwent a pulmonary flow study, the VSD was closed in $34(85 \%)$, with salvage VSD fenestration in $4(10 \%)$, and was intentionally left open in $6(15 \%)$. Survival was $85.5 \%$ at 1 year and $78.5 \%$ at 5 years. A mean PAP $\geq 25 \mathrm{~mm} \mathrm{Hg}$ was associated with worse survival $(P=.011)$. Cox regression analysis identified a mean PAP $\geq 25 \mathrm{~mm} \mathrm{Hg}$ as the sole predictor for death $(P=.037)$. Patients with an open VSD had an increased risk of reoperation $(P=.001)$ and pulmonary artery reintervention $(P=.010)$, and had a trend toward increased risk of death $(P=.059)$, compared with those with a closed VSD.

Conclusions: PAP obtained from the intraoperative pulmonary flow study is associated with medium-term survival and late RVSP in patients with PA/VSD/ MAPCAs. VSD closure for patients with a mean PAP $\geq 25 \mathrm{~mm} \mathrm{Hg}$ on a flow study is considered high risk, and sensible judgment and a low threshold for VSD fenestration are required. (J Thorac Cardiovasc Surg 2016;152:1494-503)

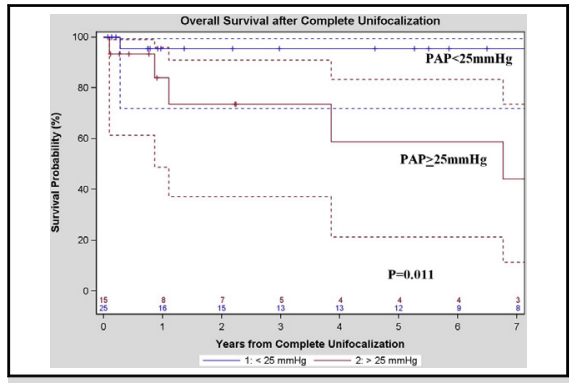

A high mean PAP $(\geq 25 \mathrm{~mm} \mathrm{Hg})$ on a flow study is associated with poor medium-term survival.

Central Message

Mean PAP predicts medium-term survival and RVSP. VSD closure in patients with PAP $\geq 25 \mathrm{~mm} \mathrm{Hg}$ should be performed with caution.

\section{Perspective}

Mean PAP on a pulmonary flow study is a strong predictor for medium-term survival and RVSP regardless of VSD status. VSD closure in patients with a mean PAP $\geq 25 \mathrm{~mm} \mathrm{Hg}$ is considered high risk, and thus sensible judgment and a low threshold for VSD fenestration are required.

See Editorial Commentary page 1504.
From the ${ }^{\mathrm{a}}$ Division of Cardiovascular Surgery and ${ }^{\mathrm{c}}$ Cardiology, The Labatt Family Heart Centre, The Hospital for Sick Children, and Department of Surgery, University of Toronto, Toronto, Ontario, Canada; ${ }^{\mathrm{b}}$ Department of Cardiothoracic Surgery, Xinhua Hospital, School of Medicine, Shanghai Jiaotong University, Shanghai, China; and ${ }^{\mathrm{d}}$ Department of Pediatrics, Faculty of Medicine \& Dentistry, University of Alberta, Edmonton, Alberta, Canada.

Read at the 95th Annual Meeting of The American Association for Thoracic Surgery, Seattle, Washington, April 25-29, 2015.

Received for publication May 4, 2015; revisions received July 24, 2016; accepted for publication July 26, 2016; available ahead of print Sept 28, 2016.

Address for reprints: Osami Honjo, MD, PhD, Division of Cardiovascular Surgery and University of Toronto, The Labatt Family Heart Centre, The Hospital for Sick Children, 555 University Ave, Toronto, Ontario, Canada, M5G 1X8 (E-mail: osami.honjo@sickkids.ca).

$0022-5223 / \$ 36.00$

Copyright $(9) 2016$ by The American Association for Thoracic Surgery

http://dx.doi.org/10.1016/j.jtcvs.2016.07.082
Pulmonary atresia (PA), ventricular septal defect (VSD), and major aortopulmonary collateral arteries (MAPCAs) pose significant surgical challenges because of the anatomic variations in the native central pulmonary artery and MAPCAs. The ultimate goal of treatment is to achieve a

Scanning this QR code will take you to the supplemental table. To view the AATS 2015 Webcast, see the URL next to the video thumbnail.

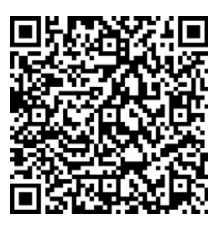




\section{Abbreviations and Acronyms \\ LVSP = left ventricular systolic pressure \\ MAPCA $=$ major aortopulmonary collateral artery \\ PA $=$ pulmonary atresia \\ PAP $=$ pulmonary artery pressure \\ $\mathrm{RV} \quad=$ right ventricular \\ RVSP = right ventricular systolic pressure \\ TNPAI $=$ total neo-pulmonary artery index \\ VSD $=$ ventricular septal defect}

biventricular in-series circulation by closing the VSD and establishing an unobstructed pathway from the right ventricle to the pulmonary vasculature with low right ventricular systolic pressure (RVSP). The unifocalization procedure for MAPCAs is a means of incorporating all available lung segments so that the VSD can be closed without significant right ventricular (RV) hypertension. Complete unifocalization has been classically achieved in a staged manner ${ }^{1-5}$ and more recently with a single-stage approach, ${ }^{6-12}$ with VSD closure rates ranging from $56 \%$ to $90 \%$. Nonetheless, the decision to perform VSD closure in patients with borderline PA and MAPCA anatomy always poses significant challenges, and the improper decision for VSD closure may be associated with high mortality and morbidity.

Prediction of the ability to close a VSD was initially attempted using an anatomic index suggested by Reddy et $a{ }^{13}$ the total neo-pulmonary artery index (TNPAI). The TNPAI was found to have an acceptable but suboptimal predictability of VSD closure because of the inability to take stenosis or kinking of MAPCAs and segmental pulmonary vascular resistance into account. ${ }^{13,14}$ Reddy et al subsequently proposed using an intraoperative pulmonary flow study as a physiological parameter to reflect total pulmonary vascular cross-sectional area and resistance after complete unifocalization. ${ }^{13-15}$ In a previous study, we showed that mean pulmonary artery pressure (PAP) on the intraoperative pulmonary flow study had better accuracy in predicting successful VSD closure compared with TNPAI or other anatomic indices. ${ }^{16}$ Nonetheless, the association between mean PAP on a pulmonary flow study and medium-term survival and RV status remain largely unknown. We hypothesized that the postoperative pulmonary vascular status and resultant RV pressure and function may be reflected by the mean PAP on a pulmonary flow study, which potentially predicts medium-term clinical outcomes of this entity.

\section{METHODS}

This retrospective study was approved by Research Ethics Board at The Hospital for Sick Children. Fifty consecutive patients with PA/VSD/ MAPCAs who underwent at least one unifocalization procedure between
January 2000 and December 2013 were identified from the surgical database. The follow-up data were collected up to October 2014. Patients with MAPCAs related to pulmonary stenosis, double-outlet right ventricle, or single-ventricle physiology were excluded. Patient profiles are shown in Table E1.

\section{Surgical Strategy and Technique}

The surgical management strategy was consistent throughout the study period. ${ }^{16}$ All patients were assessed in view of primary one-stage complete unifocalization via sternotomy at age 4 to 8 months. The staged repair was indicated in 3 circumstances: (1) presence of diminutive native branch pulmonary artery $<2 \mathrm{~mm}$, (2) presence of distal stenosis of MAPCAs inaccessible via the sternotomy approach, and (3) critical cyanosis in the neonatal period. A central shunt between the native main pulmonary artery and ascending aorta ${ }^{17}$ is typically performed in the neonatal period to rehabilitate the native pulmonary artery or to treat critical cyanosis (arterial saturation $<75 \%$ ). Unilateral unifocalization to a pericardial roll and a shunt (typically $4 \mathrm{~mm}$ ) is performed via a thoracotomy in patients with MAPCAs with distal stenosis, typically at age 2 to 3 months, followed by contralateral unifocalization or complete repair, depending on the contralateral MAPCA anatomy. Complete unifocalization after those palliative procedures is typically performed by age 8 months.

The surgical techniques for unifocalization and central pulmonary artery reconstruction and the pulmonary flow study are described in detail elsewhere. ${ }^{16}$ In brief, our strategy was to unifocalize all important MAPCAs, even those with intraparenchymal communication with other MAPCAs or the native pulmonary artery. MAPCAs were dissected beyond the area of stenosis and then anastomosed to the reconstructed central pulmonary artery without patch materials. The central pulmonary artery was reconstructed with a glutaraldehyde-treated autologous pericardial patch. A homograft patch was not used in this series. The pulmonary flow infusion was incrementally increased from 1 to $2.5 \mathrm{~L} / \mathrm{min} / \mathrm{m}^{2}$ through a cannula placed in the reconstructed pulmonary artery. A mean PAP of $2.5 \mathrm{~L} / \mathrm{min} / \mathrm{m}^{2}$ was used to determine the adequacy of VSD closure. In recent years, this was increased to $3 \mathrm{~L} / \mathrm{min} / \mathrm{m}^{2}$. The cutoff value for VSD closure during the study period was $30 \mathrm{~mm} \mathrm{Hg}$, but a more conservative approach was taken in recent years for patients with a borderline mean PAP of 25 to $30 \mathrm{~mm} \mathrm{Hg}$ who had suboptimal MAPCAs and native pulmonary artery anatomy. The RVSP was measured after VSD closure. The systemic blood pressure from a peripheral arterial line was measured and considered as left ventricular systolic pressure (LVSP). The VSD patch was fenestrated when the RVSP/LVSP ratio exceeded 0.8 . The fenestration was created in the middle of the VSD patch, which needed to be at least $5 \mathrm{~mm}$ in diameter.

\section{Data Collection}

All postoperative echocardiography reports were reviewed to assess RV function. RV dysfunction was defined when qualitatively moderate or severe RV dysfunction was noted on more than 2 consecutive echocardiograms. All preoperative angiographic images were reviewed by an investigator who was blinded to any clinical information. The TNPAI was calculated based on angiography performed just before complete unifocalization using the previously described formula. ${ }^{13}$ The follow-up RVSP and RVSP/LVSP ratio were obtained from the latest postoperative cardiac catheterization. The estimated RVSP from echocardiograms was not used. Any catheter-based interventions to the neo-pulmonary artery system, including both unifocalized MAPCAs and native pulmonary artery, and any reoperation after complete unifocalization were documented.

\section{Statistical Analysis}

Demographic, anatomic, clinical, and operative characteristics were summarized. Categorical variables are reported as frequency and percentage and compared using Fisher's exact test or the $\chi^{2}$ test, as appropriate. Continuous variables are presented as mean with standard 
deviation or median with interquartile range and were compared using Student's $t$ test. Correlations with intraoperative RVSP, intraoperative RSVP/LVSP, most recent RVSP, and most recent RVSP/LVSP were determined using Spearman's rank correlation. Associations between candidate covariates (mean PAP, number of MAPCAs, and baseline TNPAI) and the aforementioned outcomes were assessed using univariable linear or multinomial regression. Survival analysis was performed using Kaplan-Meier analysis, and survival was compared using the log-rank test. Univariable Cox proportional hazards analysis was used to assess candidate risk factors (mean PAP, number of MAPCAs, and baseline TNPAI). Outcomes assessed included death, any reintervention, operative reintervention, and catheter reintervention. A $P$ value of .05 was considered significant. All analyses were performed using SAS version 9.2 (SAS Institute, Cary, NC).

\section{RESULTS}

Demographic data for the 50 patients are presented in Table E1. At complete unifocalization, the median patient age was 11 months and median weight was $7.7 \mathrm{~kg}$. Thirty-nine patients $(78 \%)$ had a confluent native pulmonary artery, 3 patients $(6 \%)$ had a nonconfluent pulmonary artery, and 8 patients $(16 \%)$ had an absent native pulmonary artery.

\section{Surgical Strategies}

A flowchart outlining the surgical strategies is shown in Figure 1. Thirty patients $(60 \%)$ underwent one-stage unifocalization via sternotomy, and 20 patients (40\%) underwent staged unifocalization. Altogether, 47 patients (94\%) achieved complete unifocalization, and 3 patients were awaiting further unifocalization.
Among the 47 patients who achieved complete unifocalization, the VSD was closed in 34 (72.3\%) during the same procedure. Among the 30 patients who underwent one-stage unifocalization, $23(76.7 \%)$ underwent successful VSD closure, whereas $5(16.7 \%)$ required salvage VSD fenestration and $2(6.7 \%)$ had the VSD electively left open. Among 17 patients who underwent staged but complete unifocalization, VSD closure was achieved in $11(64.7 \%)$, the VSD was fenestrated in $2(11.7 \%)$, and the VSD was electively left open in $4(23.5 \%)$. There was no difference in the rate of successful VSD closure between the patients who underwent one-stage unifocalization and those who underwent staged unifocalization $(P=.246)$. Among 13 patients with a VSD electively left open, the VSD was successfully closed later in $3(23 \%)$, by surgery in 2 and by catheterization in 1 . Overall, 37 patients $(74 \%)$ achieved VSD closure by the latest follow-up.

\section{Pulmonary Flow Study and Decision Making}

The pulmonary flow study became our standard practice in 2003. Forty patients underwent a pulmonary flow study, and $10(20 \%)$ did not (6 patients before 2003, 3 patients who did not complete unifocalization, and 1 patient with favorable anatomy). All 6 patients before 2003 underwent VSD closure without a pulmonary flow study, and 3 of them $(50 \%)$ required salvage VSD fenestration owing to high RVSP.

The median PAP on the pulmonary flow study was $22 \mathrm{~mm}$ $\mathrm{Hg}$ (range, $8-44 \mathrm{~mm} \mathrm{Hg}$ ). Six patients $(15 \%)$ had a mean PAP $\geq 30 \mathrm{~mm} \mathrm{Hg}$, and in these patients the VSD was left

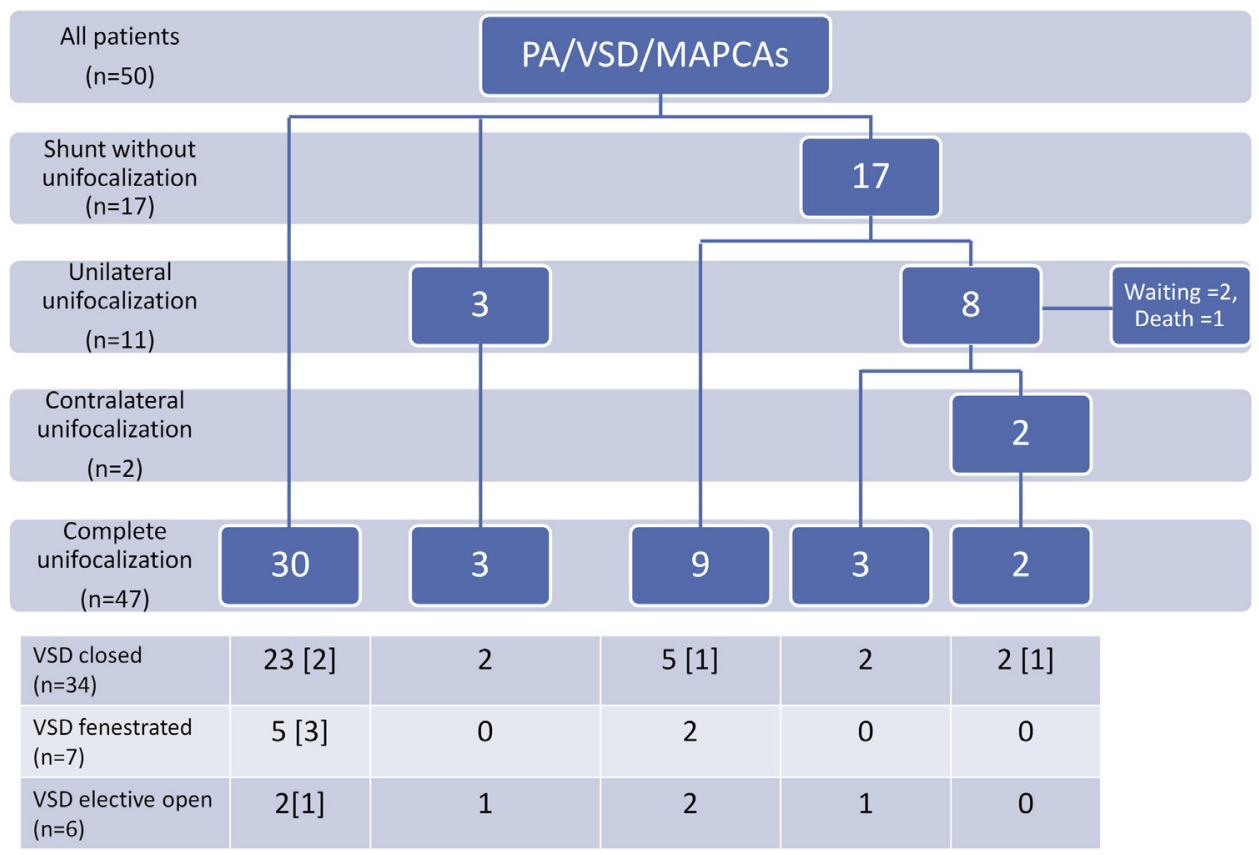

FIGURE 1. Flowchart of surgical strategy. The number in the bracket represents number of deaths. $P A$, Pulmonary atresia; $V S D$, ventricular septal defect; MAPCAs, major aortopulmonary collateral arteries. 

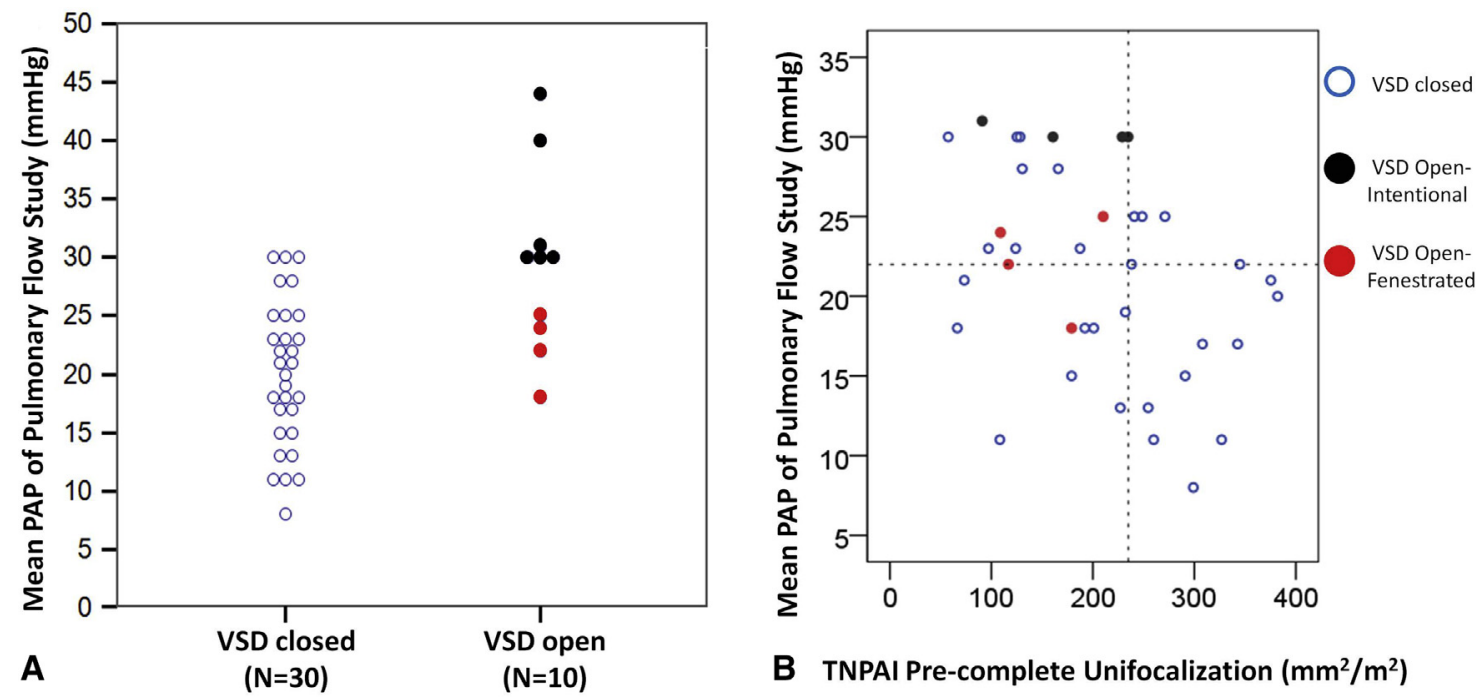

FIGURE 2. Relationship between ventricular septal defect $(V S D)$ status and mean pulmonary artery pressure $(P A P)(\mathrm{n}=40)(\mathrm{A})$ and total neo-pulmonary artery index $(T N P A I)(\mathrm{n}=38)(\mathrm{B})$.

open. The VSD was closed in 34 patients who had a mean PAP $\leq 30 \mathrm{~mm} \mathrm{Hg}$, of whom $4(11.8 \%)$ required salvage VSD fenestration owing to suprasystemic RVSP (Figure 2, $A$ ). These patients had a mean PAP of $18,22,24$, and $25 \mathrm{~mm} \mathrm{Hg}$. Patients with a low TNPAI and a high mean PAP tended to have a high likelihood of having an open VSD (Figure 2, B). The RVSP/LVSP ratio immediately following VSD closure was not correlated with mean PAP $(r=0.24 ; P=.17)$ or baseline TNPAI $(r=0.29 ; P=.09)$.

\section{Survival and Causes of Death}

The median duration of follow-up was 2.2 years (range, $0.02-14.1$ years). There were $2(4 \%)$ in-hospital deaths and $7(14 \%)$ late deaths. Causes of death are summarized in Table 1. Eight patients (89\%) completed unifocalization, and 4 patients $(44 \%)$ had the VSD closed. The median interval between the last unifocalization and death was 0.9 year (range, 1 month to 6.8 years). Causes of death included RV failure in 2 patients (both with a closed VSD), respiratory or airway compression in 2 patients, complications related to catheterization in 3 patients, sudden death in 1 patient, and palliative care in 1 patient who had Alagille syndrome and ongoing gastrointestinal bleeding. Overall, 5 deaths $(55 \%)$ were directly or indirectly related to high PAP and RVSP. Survival was $85.5 \%$ at 1 year, $82.4 \%$ at 3 years, and $78.5 \%$ at 5 years (Figure 3, A). Among the patients with complete unifocalization $(n=47)$, there was a trend toward better survival in patients with a closed VSD compared with those with an open $\operatorname{VSD}(P=.059)$.

\section{Pulmonary Flow Study and Survival}

In the 40 patients who underwent a pulmonary flow study, a mean PAP $<25 \mathrm{~mm} \mathrm{Hg}$ was associated with significantly better survival $(P=.011)$ (Figure $3, B)$. There was only 1 death (4\%; sudden death with unknown cause) among the 25 patients who had a mean PAP $<25 \mathrm{~mm} \mathrm{Hg}$. The mean PAP distribution is shown in Figure 4. Among the 30 patients who had primary VSD closure, there was a consistent trend toward an association between low PAP $(<25 \mathrm{~mm} \mathrm{Hg})$ and higher survival $(P=.039)$. When the cohort was divided into 3 groups based on mean PAP on the flow study $(<25 \mathrm{~mm} \mathrm{Hg}, 25-29 \mathrm{~mm} \mathrm{Hg}, \geq 30 \mathrm{~mm}$ $\mathrm{Hg}$ ), survival was the worst in the patients with a mean PAP of 25 to $29 \mathrm{~mm} \mathrm{Hg}(P=.008)$.

\section{Reintervention}

Seven patients (14.8\%) underwent reoperation, including VSD closure in 2 patients, right ventricle-pulmonary artery conduit replacement in 3 patients (with concomitant VSD closure in 1 patient), relief of bronchus compression in 2 patients, pulmonary arterioplasty in 1 patient, and RV outflow tract aneurysm repair (with concomitant VSD closure) in 1 patient. The freedom from reoperation was $93.4 \%$ at 1 year, $78.1 \%$ at 3 years, and $78.1 \%$ at 5 years. Catheter reintervention for the neo-pulmonary artery system was required in 27 patients $(54 \%)$ after complete unifocalization. Overall freedom from catheter-based pulmonary artery reintervention was $63.3 \%$ at 1 year, $36.2 \%$ at 3 years, and $29.6 \%$ at 5 years.

\section{Right Ventricle}

Four out of 49 early survivors $(8.2 \%)$ developed moderate $(n=2)$ or severe $(n=2) R V$ systolic dysfunction. The freedom from moderate/severe RV systolic dysfunction was $89.8 \%$ at 1 year, $89.8 \%$ at 3 years, and $82.3 \%$ at 5 years. The VSD status of 4 patients was closed in 3 patients and fenestrated in 1 patient. One patient died due 
TABLE 1. Causes of death

\begin{tabular}{|c|c|c|c|c|c|c|c|c|c|c|c|}
\hline Patient & $\begin{array}{l}\text { Unifoca- } \\
\text { lization } \\
\text { strategy }\end{array}$ & $\begin{array}{l}\text { Complete } \\
\text { unifoca- } \\
\text { lization } \\
\end{array}$ & $\begin{array}{c}\text { Age at } \\
\text { complete } \\
\text { unifoca- } \\
\text { lization, } y\end{array}$ & $\begin{array}{c}\text { VSD } \\
\text { status }\end{array}$ & $\begin{array}{c}\text { Mean PAP } \\
\text { of flow } \\
\text { study, } \\
\text { mm Hg } \\
\end{array}$ & $\begin{array}{c}\text { RVSP/ } \\
\text { LVSP } \\
\text { during } \\
\text { operation }\end{array}$ & $\begin{array}{c}\text { In- } \\
\text { hospital } \\
\text { death }\end{array}$ & $\begin{array}{c}\text { Follow-up } \\
\text { RVSP/ } \\
\text { LVSP } \\
\end{array}$ & $\begin{array}{c}\text { Interval } \\
\text { after } \\
\text { complete } \\
\text { unifoca- } \\
\text { lization } \\
\end{array}$ & Cause of death & $\begin{array}{c}\text { Category } \\
\text { of death } \\
\end{array}$ \\
\hline 10 & One-stage & Yes & 0.6 & $\begin{array}{l}\text { Open } \\
\text { (fen) }\end{array}$ & NA & $>1$ & No & 0.70 & $0.9 \mathrm{y}$ & $\begin{array}{l}\text { LAD thrombosis during } \\
\text { attempted VSD } \\
\text { device closure }\end{array}$ & $\begin{array}{r}\text { Catheter- } \\
\text { related }\end{array}$ \\
\hline 12 & One-stage & Yes & 0.7 & $\begin{array}{l}\text { Open } \\
\quad(\text { fen })\end{array}$ & NA & $>1$ & No & NA & $2 \mathrm{mo}$ & $\begin{array}{l}\text { Rupture of RVOT } \\
\text { conduit pseudo- } \\
\text { aneurysm from } \\
\text { autopsy }\end{array}$ & $\begin{array}{r}\text { Sudden } \\
\text { death }\end{array}$ \\
\hline 25 & One-stage & Yes & 0.9 & Closed & 28 & 0.79 & No & 0.97 & $3.9 \mathrm{y}$ & $\begin{array}{l}\text { Severely reduced RV } \\
\text { function; MOF } \\
\text { triggered by } \\
\text { respiratory infection }\end{array}$ & Heart \\
\hline 28 & Staged & Yes & 0.6 & Closed & 23 & 0.47 & No & NA & $3 \mathrm{mo}$ & $\begin{array}{l}\text { Unknown, outside } \\
\text { hospital. The patient } \\
\text { had postoperative } \\
\text { bronchus } \\
\text { compression before } \\
\text { discharge }\end{array}$ & Airway \\
\hline 40 & One-stage & Yes & 0.4 & Open & 31 & NA & No & 1.06 & $6.8 \mathrm{y}$ & $\begin{array}{l}\text { Possible pulmonary } \\
\text { hemorrhage after } \\
\text { balloon dilation of } \\
\text { pulmonary artery }\end{array}$ & $\begin{array}{r}\text { Catheter- } \\
\text { related }\end{array}$ \\
\hline 41 & One-stage & Yes & 0.6 & Closed & 25 & 0.45 & No & 0.74 & $0.9 \mathrm{y}$ & $\begin{array}{l}\text { Cardiac arrest the day } \\
\text { after a catheterization } \\
\text { procedure for } 3 / 4 \\
\text { RVSP/LVSP }\end{array}$ & $\begin{array}{l}\text { Catheter- } \\
\text { related }\end{array}$ \\
\hline 42 & Staged & No & 1.5 & Open & NA & NA & No & NA & $6 \mathrm{mo}$ & $\begin{array}{l}\text { Palliative care for } \\
\text { other disease }\end{array}$ & Other \\
\hline 43 & Staged & Yes & 1 & Closed & 30 & 0.63 & Yes & 0.77 & $1.1 \mathrm{y}$ & $\begin{array}{l}\text { Worsening diastolic } \\
\text { function, hypoxia, } \\
\text { infection, airway } \\
\text { compression in } \\
\text { early stage }\end{array}$ & Heart \\
\hline 45 & One-stage & Yes & 0.5 & $\begin{array}{l}\text { Open } \\
\quad \text { (fen) }\end{array}$ & 25 & $>1$ & Yes & NA & $1 \mathrm{mo}$ & $\begin{array}{l}\text { Bronchus compression, } \\
\text { respiratory failure }\end{array}$ & Airway \\
\hline
\end{tabular}

$\overline{V S D}$, Ventricular septal defect; $P A P$, pulmonary artery pressure; $R V S P$, right ventricular systolic pressure; $L V S P$, left ventricular systolic pressure; fen, fenestrated; $N A$, not available; $L A D$, left anterior descending; $R V O T$, right ventricular outflow tract; $R V$, right ventricular; $M O F$, multiorgan failure.

to RV dysfunction, and 1 patient had persistent moderate $\mathrm{RV}$ dysfunction. RV function recovered to normal in 2 patients. One patient with normal RV systolic function developed significant RV diastolic dysfunction with restrictive lung disease, leading to death. Predictive analysis for RV dysfunction was not possible owing to the small number of events.

Follow-up RVSP/LVSP data were available from postoperative catheterization in 17 of the 30 patients $(57 \%)$ who underwent VSD closure. The mean PAP was correlated with the latest RVSP/LVSP ratio $(r=046$; $P=.02$ ) (Figure 5, A), and the patients who experienced late death appeared to have a high RVSP/LVSP ratio (all
$>0.7$ ). The latest RVSP/LVSP ratio was inversely correlated with baseline TNPAI $(r=0.40 ; P=.05)$ (Figure 5B). Among the 41 medium-term survivors, New York Heart Association class was class I in 35 patients $(85.4 \%)$, class II in 5 patients $(12.2 \%)$, and class III in 1 patient $(2.4 \%)$.

\section{Risk Factor Analysis}

Univariate Cox regression identified mean PAP on the pulmonary flow study as a marginal predictor for survival (hazard ratio [HR], 1.1; 95\% confidence interval [CI], $1-1.2 ; P=.06$; with square root transformation: HR, 2.9; $95 \%$ CI, 0.99-8.54; $P=.06$ ). A mean PAP $\geq 25 \mathrm{~mm} \mathrm{Hg}$ 


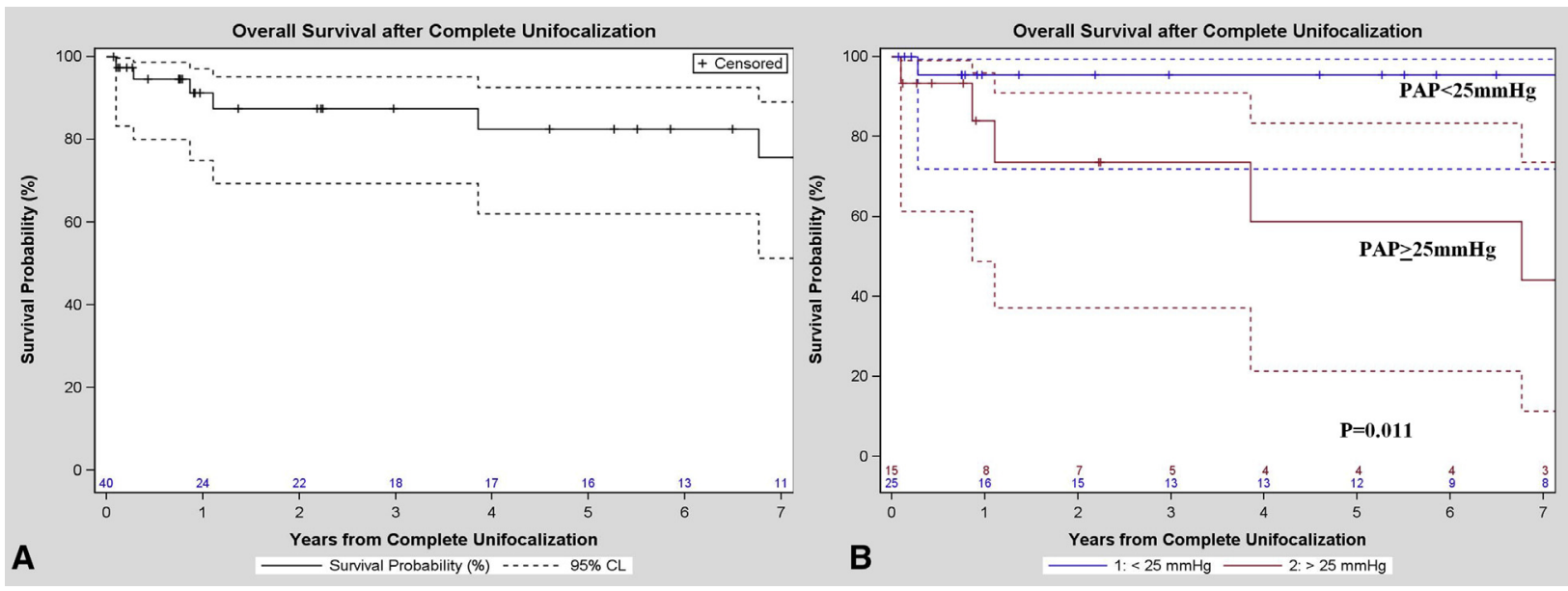

FIGURE 3. Overall survival (A) and survival stratified by mean pulmonary artery pressure $(P A P)$ on the flow study (B).

predicted worse survival (HR, 9.804; 95\% CI, 1.143-83.3; $P=.037)$. Other factors, including TNPAI, age, weight, RVSP/LVSP, and unifocalization strategy, were not predictive of survival $(P>.10$ for all). No predictive value was identified for any reintervention, reoperation, or RV dysfunction.

\section{DISCUSSION}

The most important result from this study is that the physiological condition of the pulmonary vasculature after complete unifocalization, as estimated by mean PAP on the pulmonary flow study, had a significant impact on medium-term survival. There was a striking difference in survival between the patients with a mean PAP $<25 \mathrm{~mm}$ $\mathrm{Hg}$ and those with a mean PAP $\geq 25 \mathrm{~mm} \mathrm{Hg}$. Regardless of native pulmonary artery and MAPCA anatomy, the patients with a mean PAP $<25 \mathrm{~mm} \mathrm{Hg}$ had very favorable medium-term survival, with no operative deaths and only 1 non-cardiac-related late death $(4 \%)$. The subgroup of patients with a mean PAP of 25 to $29 \mathrm{~mm} \mathrm{Hg}$ had the worst medium-term survival, owing in part to high-risk VSD closure in the borderline patients. This study underscores the importance of efforts aimed at incorporating all available MAPCAs and subsequent lung segments to retain as much of the pulmonary vascular bed as possible, which is the major determinant of medium-term clinical outcomes. Our findings also indicate that a more appropriate cutoff mean PAP on the pulmonary flow study appears to be $25 \mathrm{~mm} \mathrm{Hg}$ rather than $30 \mathrm{~mm} \mathrm{Hg}$.

\section{Causes of Death}

The causes of death in 9 patients illustrate the challenges following complete unifocalization and also provide important insight into how we should manage those patients at the time of complete unifocalization and VSD closure. Causes of death included RV dysfunction, airway compression, respiratory-related, and acute deaths related to catheter reintervention. d'Udekem et $\mathrm{al}^{5}$ reported similar causes of death in their long-term follow-up study. Two deaths were directly related to RV dysfunction (1 biventricular systolic dysfunction, 1 biventricular diastolic dysfunction). Both patients underwent high-risk VSD closure with a mean PAP of 28 and $30 \mathrm{~mm} \mathrm{Hg}$, respectively. Although there are many survivors with a mean PAP $\geq 25 \mathrm{~mm} \mathrm{Hg}$ in our series, early fenestration of the VSD could have been considered for those patients at the onset of signs of RV dysfunction. The causes of terminal events differed, but 5 patients clearly died from pulmonary artery and RV hypertension. Major airway compression was also common in the patients who died (3 patients; 33\%), which is not surprising given that a pressurized conduit or reconstructed pulmonary artery can compress the already vulnerable airway. ${ }^{13,18,19}$ We did not find many modifiable factors that could have prevented death; however, we should at least have a careful follow-up plan for assessing RV performance in patients who undergo high-risk VSD closure with persistent RV hypertension.

\section{Relevance of Pulmonary Flow Studies}

Four patients $(10 \%)$ had a false-negative pulmonary flow study, which led to salvage VSD fenestration. The mean PAP of these 4 patients was $18,22,24$, and $25 \mathrm{~mm} \mathrm{Hg}$, respectively. One patient had significant left pulmonary artery stenosis caused by conduit replacement, which was unrecognized at the time of the decision to perform VSD fenestration. This patient later underwent successful VSD closure. The reasons for the false-negative studies in the other 3 patients are not clear. Certain factors are known to potentially cause a false-positive study, including inadequate left atrial decompression, or incomplete lung recruitment; however, we did not identify any significant false- positive study in this series. One of the most 


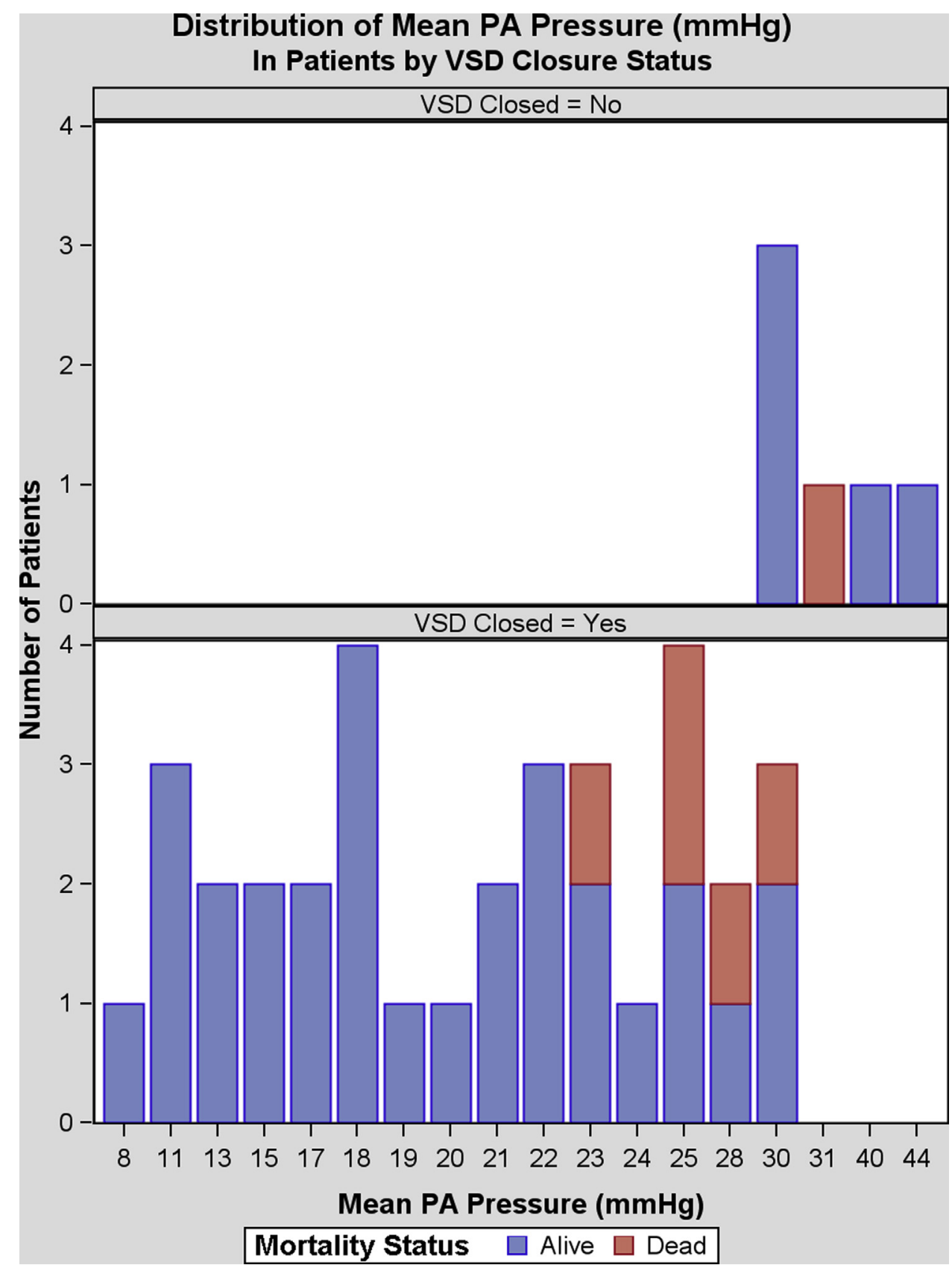

FIGURE 4. Histogram demonstrating distribution of mean pulmonary artery pressure $(P A P)$ on the flow study and death at any point during the study. $V S D$, Ventricular septal defect.

important results of our analysis is that the optimal cutoff value for VSD closure appears to be $25 \mathrm{~mm} \mathrm{Hg}$ rather than $30 \mathrm{~mm} \mathrm{Hg}$. We still believe that some VSDs would be closable in patients with a mean PAP of 25 to $30 \mathrm{~mm}$ $\mathrm{Hg}$, but sensible judgment is required for this high-risk subgroup, with a low threshold to perform VSD fenestration if necessary. Our group has been exploring the potential of cardiac magnetic resonance imaging studies with flow measurements to predict the ability to close the VSD and predict subsequent RVSP after VSD closure. ${ }^{20}$ Based on a preliminary study, there appears to be a correlation between preoperative total pulmonary blood flow on cardiac magnetic resonance imaging and postoperative RVSP, although the data are not as definitive as for mean PAP.

\section{Reintervention for the Neo-Pulmonary Artery System}

Reintervention for the neo-pulmonary artery system after complete unifocalization was common in this and other studies. ${ }^{18,21}$ Freedom from pulmonary artery 

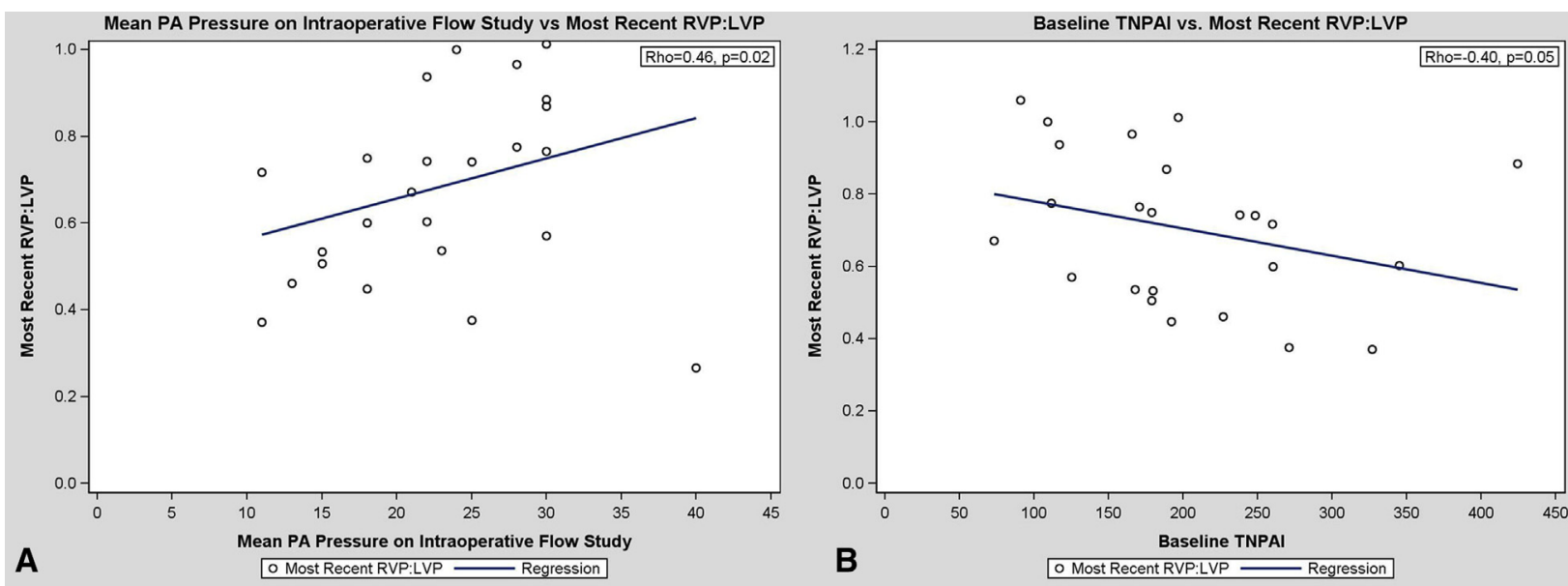

FIGURE 5. Correlation between the latest right ventricular systolic pressure/left ventricular systolic pressure (RVSP/LVSP) ratio and mean pulmonary artery pressure $(P A P)$ on the flow study (A) or baseline total neo-pulmonary artery index $(T N P A I)(\mathrm{B})(\mathrm{n}=17)$.

reintervention was $36.2 \%$ at 3 years, which is not surprising given our practice of unifocalizing all available MAPCAs including very small or stenotic ones, as well as the tendency toward progressive stenosis in unifocalized MAPCAs. The patients with an open VSD required significantly more pulmonary artery reinterventions, which reflects poor MAPCA quality in this subgroup and also the efforts to render these patients candidates for future VSD closure. Of note, catheter intervention for those patients with high PAP and RVSP is not a benign procedure. Three late deaths $(33 \%)$ in our series occurred during or after catheter intervention, and this intervention carries a high risk of pulmonary hemorrhage and pulmonary artery dissection, rupture, or pseudoaneurysm formation.

\section{Strategy for Patients With an Open VSD}

The patients with an open VSD as a result of a high mean PAP and/or RVSP undoubtedly represent the most vulnerable subgroup with the highest mortality and morbidity. Only a minority $(n=3 ; 23 \%)$ of the patients with an open VSD subsequently underwent VSD closure after rehabilitation of a peripheral pulmonary artery. There are limited surgical and medical options for patients with very poor MAPCA and native pulmonary artery size and quality, and only palliative catheter interventions were provided to this subgroup after unifocalization and shunt placement. Theoretically, heart and lung transplantation could be considered, ${ }^{22}$ although we did not consider or evaluate those patients for heart and lung transplantation during the study period.

\section{Study Limitations}

The major limitation of this study is its retrospective nature and relatively small cohort in a single institution. We were not able to perform repeated-events analysis owing to the small sample size. A second limitation is the relatively short follow-up period.

\section{CONCLUSIONS}

The mean PAP obtained from the pulmonary flow study is predictive of medium-term survival and late RVSP. Patients with a mean PAP $<25 \mathrm{~mm} \mathrm{Hg}$ had very high medium-term survival regardless of pulmonary artery and MAPCA anatomy. VSD closure for patients with a mean PAP $\geq 25 \mathrm{~mm}$ $\mathrm{Hg}$ is considered high risk, and sensible judgment and a low threshold for VSD fenestration are required.

\section{Webcast}

You can watch a Webcast of this AATS meeting presentation by going to: http://webcast.aats.org/2015/Video/ Wednesday/04-29-15_612_0731_Zhu.mp4.

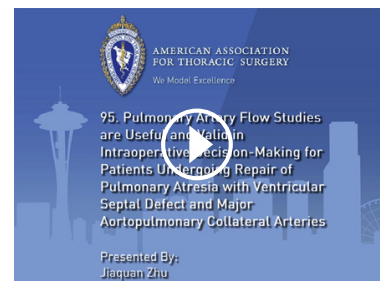

\section{Conflict of Interest Statement}

Authors have nothing to disclose with regard to commercial support.

\section{References}

1. Puga FJ, Leoni FE, Julsrud PR, Mair DD. Complete repair of pulmonary atresia, ventricular septal defect, and severe peripheral arborization abnormalities of the central pulmonary arteries: experience with preliminary unifocalization procedures in 38 patients. J Thorac Cardiovasc Surg. 1989;98: 1018-28; discussion 28-9.

2. Iyer KS, Mee RB. Staged repair of pulmonary atresia with ventricular septal defect and major systemic to pulmonary artery collaterals. Ann Thorac Surg. 1991;51:65-72. 
3. Yagihara T, Yamamoto F, Nishigaki K, Matsuki O, Uemura H, Isizaka T, et al. Unifocalization for pulmonary atresia with ventricular septal defect and major aortopulmonary collateral arteries. J Thorac Cardiovasc Surg. 1996;112: 392-402.

4. Duncan BW, Mee RB, Prieto LR, Rosenthal GL, Mesia CI, Qureshi A, et al. Staged repair of tetralogy of Fallot with pulmonary atresia and major aortopulmonary collateral arteries. J Thorac Cardiovasc Surg. 2003;126: 694-702.

5. d'Udekem Y, Alphonso N, Nørgaard MA, Cochrane AD, Grigg LE, Wilkinson JL, et al. Pulmonary atresia with ventricular septal defects and major aortopulmonary collateral arteries: unifocalization brings no long-term benefits. J Thorac Cardiovasc Surg. 2005;130:1496-502.

6. Tchervenkov CI, Salasidis G, Cecere R, Béland MJ, Jutras L, Paquet M, et al. One-stage midline unifocalization and complete repair in infancy versus multiple-stage unifocalization followed by repair for complex heart disease with major aortopulmonary collaterals. J Thorac Cardiovasc Surg. 1997;114: 727-35; discussion 35-7.

7. Carotti A, Di Donato RM, Squitieri C, Guccione P, Catena G. Total repair of pulmonary atresia with ventricular septal defect and major aortopulmonary collaterals: an integrated approach. J Thorac Cardiovasc Surg. 1998;116:914-23.

8. McElhinney DB, Reddy VM, Hanley FL. Tetralogy of Fallot with major aortopulmonary collaterals: early total repair. Pediatr Cardiol. 1998;19:289-96.

9. Murthy KS, Krishnanaik S, Coelho R, Punnoose A, Arumugam SB, Cherian KM Median sternotomy single-stage complete unifocalization for pulmonary atresia, major aorto-pulmonary collateral arteries and VSD-early experience. Eur J Cardiothorac Surg. 1999;16:21-5.

10. Reddy VM, Liddicoat JR, Hanley FL. Midline one-stage complete unifocalization and repair of pulmonary atresia with ventricular septal defect and major aortopulmonary collaterals. J Thorac Cardiovasc Surg. 1995;109: 832-44; discussion 44-5.

11. Davies B, Mussa S, Davies P, Stickley J, Jones TJ, Barron DJ, et al. Unifocalization of major aortopulmonary collateral arteries in pulmonary atresia with ventricular septal defect is essential to achieve excellent outcomes irrespective of native pulmonary artery morphology. J Thorac Cardiovasc Surg. 2009; 138:1269-75.e1

12. Brawn WJ, Jones T, Davies B, Barron D. How we manage patients with major aorta pulmonary collaterals. Semin Thorac Cardiovasc Surg Pediatr Card Surg Апnu. 2009;12:152-7.

13. Reddy VM, Petrossian E, McElhinney DB, Moore P, Teitel DF, Hanley FL. One-stage complete unifocalization in infants: when should the ventricular septal defect be closed? J Thorac Cardiovasc Surg. 1997;113:858-66; discussion 866-8.

14. Carrillo SA, Mainwaring RD, Patrick WL, Bauser-Heaton HD, Peng L, Reddy VM, et al. Surgical repair of pulmonary atresia with ventricular septal defect and major aortopulmonary collaterals with absent intrapericardial pulmonary arteries. Ann Thorac Surg. 2015;100:606-14.

15. Mainwaring RD, Sheikh AY, Punn R, Reddy VM, Hanley FL. Surgical outcomes for patients with pulmonary atresia/major aortopulmonary collaterals and Alagille syndrome. Eur J Cardiothorac Surg. 2012;42:235-40; discussion 40-1.

16. Honjo O, Al-Radi OO, MacDonald C, Tran KC, Sapra P, Davey LD, et al. The functional intraoperative pulmonary blood flow study is a more sensitive predictor than preoperative anatomy for right ventricular pressure and physiologic tolerance of ventricular septal defect closure after complete unifocalization in patients with pulmonary atresia, ventricular septal defect, and major aortopulmonary collaterals. Circulation. 2009;120(11 Suppl):S46-52.

17. Watterson KG, Wilkinson JL, Karl TR, Mee RB. Very small pulmonary arteries: central end-to-side shunt. Ann Thorac Surg. 1991;52:1132-7.

18. Reddy VM, McElhinney DB, Amin Z, Moore P, Parry AJ, Teitel DF, et al. Early and intermediate outcomes after repair of pulmonary atresia with ventricular septal defect and major aortopulmonary collateral arteries: experience with 85 patients. Circulation. 2000;101:1826-32.

19. Schulze-Neick I, Ho SY, Bush A, Rosenthal M, Franklin RC, Redington AN, et al. Severe airflow limitation after the unifocalization procedure: clinical and morphological correlates. Circulation. 2000;102(19 Suppl 3):III142-7.

20. Grosse-Wortmann L, Yoo SJ, van Arsdell G, Chetan D, Macdonald C, Benson L, et al. Preoperative total pulmonary blood flow predicts right ventricular pressure in patients early after complete repair of tetralogy of Fallot and pulmonary atresia with major aortopulmonary collateral arteries. J Thorac Cardiovasc Surg. 2013; $146: 1185-90$.

21. Carotti A, Albanese SB, Filippelli S, Ravà L, Guccione P, Pongiglione G, et al. Determinants of outcome after surgical treatment of pulmonary atresia with ventricular septal defect and major aortopulmonary collateral arteries. J Thorac Cardiovasc Surg. 2010;140:1092-103.

22. Januszewska K, Malec E, Juchem G, Kaczmarek I, Sodian R, Uberfuhr P, et al. Heart-lung transplantation in patients with pulmonary atresia and ventricular septal defect. J Thorac Cardiovasc Surg. 2009;138:738-43.

Key Words: PA/VSD/MAPCAs, pulmonary flow study, unifocalization

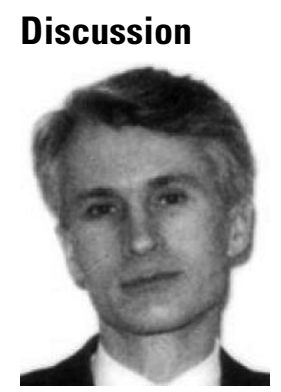

Dr Hanley (Stanford, Calif). This was a very nice presentation. I also appreciate you providing the manuscript in a timely fashion, which I was also able to review. It's very gratifying to see this kind of a study, which objectively shows the benefit of the flow study. As you may well know, and as you referenced, when we first designed the flow study in 1997, it was because of the unpredictable hemodynamics observed after closing the VSD. During the development of the flow study, the initial studies were done in the laboratory, so it wasn't clear exactly what flow study hemodynamic thresholds would prove to adequately predict safe pulmonary artery pressures after intracardiac repair in the clinical setting. We initially used a flow rate of $2.5 \mathrm{~L} /$ minute $/ \mathrm{m}^{2}$, as you're using in this study, and a cutoff pressure of 25 to $30 \mathrm{~mm} \mathrm{Hg}$. Very quickly after that, within 2 or 3 years, we adjusted these thresholds and started using a flow rate of $3 \mathrm{~L}$ and an absolute cutoff of $25 \mathrm{~mm} \mathrm{Hg}$. So if you put all of that together, with higher flow rates and lower pressure cutoff, we're looking at a much stricter threshold for pulmonary vascular resistance. We moved to the stricter thresholds because we initially found that some patients ended up with right ventricle:left ventricle pressure ratios $>0.5$ after intracardiac repair using the original thresholds. So using the stricter thresholds, the incidence of complete repair at the time of the unifocalization is actually lower. And we've come to the conclusion that, although there is urgency to unifocalizing, there is no urgency to doing the complete repair. The point of early intervention is to salvage the collaterals, and the full repair can wait until the vasculature is ready.

It seems one of the main findings from your study is that that the 25 to 30 pressure range is riskier. We would completely agree with that, and it's a very nice objective contribution that I think should be widely adopted.

We don't use the flow study in every single patient. There is an occasional patient, like an infant who is in very-high-output heart failure, essentially acting physiologically like a truncus, with large unobstructed collaterals, in whom we do not find it necessary to perform 
the flow study and in whom we would go right to intracardiac repair after the unifocalization is completed. So in our last 500 or so MAPCA patients, we've used the flow study about $60 \%$ of the time.

So that brings me to my first question. When a patient fails a flow study, how do you manage that patient in terms of pulmonary blood flow? Do you put on a right ventricle-to-pulmonary artery conduit and leave the VSD open, or do you do a shunt?

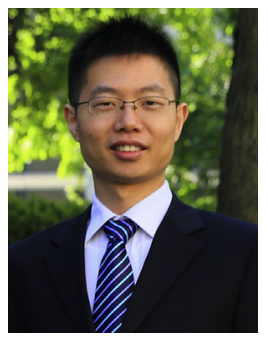

Dr Zhu (Toronto, Ontario, Canada). Thank you for your comments. That's a very good question. In our center, in the early years of this series, we preferred to place an RV-PA conduit, but in recent years we have an evolving strategy to place a BT shunt.

I think there are several potential advantages to a RV-PA conduit. As we show in this study, those patients who had VSD open tend to have more catheter-related reintervention of the neo pulmonary artery system, and it's easier to get access to balloon dilate those PA through a right ventricle-pulmonary artery conduit.

The second advantage of a right ventricle-pulmonary artery conduit is that in some patients, we left the VSD fenestrated. There is a chance of closing the VSD by a device later, which can avoid another sternotomy. However, in recent years we have found some complications with this strategy. We had 2 patients who developed $\mathrm{RV}$ outflow tract aneurysm, 1 patient died, and 1 patient had successful surgery. We think that this is due to the high RV pressure and the pulmonary artery pressure. So now we are changing our strategy and are moving to place a BT shunt.

Dr Hanley. I would completely agree with that. For a long time now, we have used a BT shunt in patients with a failed flow study for some of the reasons you just mentioned. Systemic level pressure is present in the conduits by definition, and we found an unacceptably high incidence of aneurysms when right ventricle-pulmonary artery conduits were used. Also, we noticed reduced right ventricular function in the setting of a ventriculotomy with the right ventricle at systemic level pressure. Another reason, and maybe most importantly, you don't nurture the pulmonary vascular bed as well in that subset of patients with a conduit, because you have uncontrolled blood flow into that conduit. If you put a BT shunt in, you can regulate the pressure and the flow into the vascular bed to allow the ideal hemodynamic environment for vascular growth. This maximizes the number of patients with initial failed flow studies who will get to the point where you can close the VSD. So our current philosophy is, don't touch the right ventricle and don't go in the heart, unless you're going to do a full repair with a pretty much guaranteed low RV pressure.

Dr Zhu. I completely agree with that.

Dr Hanley. My second question is: When you do your full repairs, how do you perform your follow-up?

Dr Zhu. Basically we have a regular follow-up in the clinic with echocardiography. If echocardiography shows increased RV systolic pressure-for example, reaching more than two-thirds systemic pressure-we would like to take the patient back to the cath lab and then see whether there is any distal pulmonary artery problem. And if a patient had a VSD left open, we also had regular follow-up of the catheterization, because we know that we need to do something to rehabilitate the neo-PA system. Also, in some patients we might have a chance to close the VSD by a device. Actually, we have had 2 patients who had a VSD closed by a device, but 1 of them died of some complication.

Dr Hanley. My final question. So, based on the data from this study, are you readjusting your thresholds for the flow study?

Dr Zhu. That's a very good question. Currently we also use your strategy of the new flow study in patients with this borderline mean PAP; we increase the flow to $3 \mathrm{~L}$. However, if the flow is $3 \mathrm{~L}$ and the PAP is between 25 and $30 \mathrm{~mm} \mathrm{Hg}$, we would consider the preoperative morphological data. If the MAPCA anatomy looks good, and the operation is fine, we would still try to provide an opportunity to close the VSD, because we know that if we fail, we can still fenestrate. However, if we have any concerns, we like to leave the VSD open.

Dr Hanley. I would just want to make one final point, to again emphasize the urgency in the timing to get the full unifocalization and get the collaterals out of the systemic circulation. That's the way to get the best pulmonary vascular bed. Any urgency to do a complete repair is a false one. We all feel better as surgeons when we do a complete repair all at one stage, and the family is happy. But we have become much more reticent and much more cautious about doing the intracardiac part of it unless we're assured that we're going to have an RV pressure that's half systemic or less. Eventually you can get to a full repair with low pressure in the majority of patients, but that requires deferring the intracardiac stage in the appropriate cases.

Dr Zhu. That's a very good point, and we will consider that. Thank you. 
TABLE E1. Demographic data

\begin{tabular}{|c|c|}
\hline Characteristic & Value \\
\hline Number of patients & 50 \\
\hline Males, n (\%) & $25(50)$ \\
\hline Females, n $(\%)$ & $25(50)$ \\
\hline Age at complete unifocalization, mo, median (range) & $11(1-194.4)$ \\
\hline Weight at complete unifocalization, $\mathrm{kg}$, median (range) & $7.7(2.7-64.9)$ \\
\hline \multicolumn{2}{|l|}{ Native pulmonary artery anatomy, n (\%) } \\
\hline Confluent and well-developed & $22(44)$ \\
\hline Confluent and diminutive & $17(34)$ \\
\hline Nonconfluent & $3(6)$ \\
\hline Absent & $8(16)$ \\
\hline One-stage complete unifocalization, n ( $\%)$ & $30(60)$ \\
\hline Staged unifocalizations, n (\%) & $20(40)$ \\
\hline Central shunt & $17(34)$ \\
\hline Unilateral unifocalization from thoracotomy & $9(18)$ \\
\hline Bilateral unifocalization from thoracotomy & $2(4)$ \\
\hline Sternotomy for complete unifocalization & $17(34)$ \\
\hline Right ventricle-pulmonary artery connection, n (\%) & 47 \\
\hline Valved conduit & $43(91.5)$ \\
\hline Central shunt & $2(4.3)$ \\
\hline Valved conduit + central shunt & $1(2.1)$ \\
\hline Transannular patch & $1(2.1)$ \\
\hline MAPCAs, n (\%) & 182 \\
\hline Unifocalized & $143(78.6)$ \\
\hline Ligated & $33(18.1)$ \\
\hline Waiting for unifocalization & $2(1.1)$ \\
\hline Not unifocalized & $4(2.2)$ \\
\hline \multicolumn{2}{|l|}{ Associated operations, n (\%) } \\
\hline Coarctation & $2(4)$ \\
\hline Left pulmonary vein stenosis repair & $1(2)$ \\
\hline Supra mitral ring repair & $1(2)$ \\
\hline
\end{tabular}

MAPCAs, Major aortopulmonary collateral arteries. 